\title{
Suppression of non-Poissonian shot noise by Coulomb correlations in ballistic conductors
}

\author{
O. M. Bulashenko and J. M. Rubí \\ Departament de Física Fonamental, Universitat de Barcelona, Diagonal 647, E-08028 Barcelona, Spain \\ V. A. Kochelap \\ Department of Theoretical Physics, Institute of Semiconductor Physics, Kiev 252028, Ukraine
}

(Received 29 November 1999; revised manuscript received 15 May 2000)

\begin{abstract}
We investigate the current injection into a ballistic conductor under the space-charge limited regime, when the distribution function of injected carriers is an arbitrary function of energy $F_{c}(\varepsilon)$. The analysis of the coupled kinetic and Poisson equations shows that the injected current fluctuations may be essentially suppressed by Coulomb correlations, and the suppression level is determined by the shape of $F_{c}(\varepsilon)$. This is in contrast to the time-averaged quantities: the mean current and the spatial profiles are shown to be insensitive to $F_{c}(\varepsilon)$ in the leading-order terms at high biases. The asymptotic high-bias behavior for the energy resolved shot-noise suppression has been found for an arbitrary (non-Poissonian) injection, which may suggest a new field of investigation on the optimization of the injection energy profile to achieve the desired noisesuppression level.
\end{abstract}

\section{INTRODUCTION}

Randomness in the transmission of discrete charge carriers in mesoscopic conductors leads to the fluctuations of the electric current called shot noise. ${ }^{1,2}$ Recently, shot-noise measurements are emerging as an important tool to probe carrier interactions in mesoscopic systems. ${ }^{3}$ As interactions between electrons can regulate their motion, this effect may be detected in the shot-noise reduction, but cannot be deduced from time-averaged dc measurements. Usually, the shot-noise level is said to be reduced when its spectral density is lower in respect to the Poissonian value $S_{I}^{\text {Poisson }}$ $=2 q I$, which is characteristic for transmission of uncorrelated carriers. (Here, $q$ is the electron charge and $I$ is the mean current.) The sub-Poisson shot noise could arise due to the Pauli exclusion principle or Coulomb interactions. The diversity of examples is available from recent reviews. ${ }^{1,2}$

A matter of particular interest is the significance of Coulomb interactions in scattering-free or ballistic conductors. ${ }^{4-7}$ This subject is important not only from a fundamental, but also from an applied point of view. Indeed, as the dimensions of practical electronic devices are scaled down, the ballistic component in carrier motion becomes dominant. ${ }^{8}$ Then, unavoidable electric charge of carriers and their redistribution across the device both give rise to the charge-limited ballistic transport. Based on ballistic transport, a variety of new electronic devices is currently discussed in view of future applications to ultralarge scale integrated circuits, logic, and memory technology, ${ }^{9-12}$ and new experimental techniques, like ballistic electron emission spectroscopy, have already been realized. ${ }^{13,14}$ In chargelimited ballistic conductors the shot-noise measurements may become one of the major tools not only to identify the ballistic transport, but also to probe carrier interactions and other electronic properties.

In the absence of scattering, the transport and noise properties of ballistic conductors are determined, to a great extent, by the contacts (emitters). When the injecting contact is in a local equilibrium and the electron density injected into a ballistic conductor is low, the electron gas is nondegenerate and described by the Maxwell-Boltzmann distribution function. In this case, the injected electrons are statistically independent obeying the Poissonian statistics. The self-consistent theory of shot-noise suppression due to Coulomb interactions for this type of ballistic injection has been recently developed. ${ }^{7}$ However, in nanoscale devices the injected carriers may be degenerate, or income from an emitter with an extremely nonequilibrium distribution, like in a hot-electron transistor, resonant-tunneling-diode emitter, superlattice emitter, etc. (see, e.g., Refs. 15-19). The incoming carriers may be correlated a priori and follow non-Poissonian statistics.

The main purpose of the present paper is to develop a self-consistent theory of shot noise in two-terminal ballistic space-charge-limited conductors with an arbitrary injection energy distribution $F_{c}(\varepsilon)$, which would also be valid for any given correlation properties of injected carriers. To distinguish the pure effect of Coulomb interactions on the shotnoise suppression, it will be convenient to measure the noisesuppression level in respect to the shot noise of nonPoissonian flow with disregarded Coulomb interactions, rather than to the Poissonian $2 q I$ value. We have derived the analytical formulas that determine the steady-state and noise characteristics in ballistic conductors under the action of Coulomb interactions in the asymptotic limit of high biases. The time-averaged quantities are found to be insensitive to $F_{c}(\varepsilon)$ in the leading-order terms, giving, in particular, the universal Child law for the mean current. In contrast, the current noise is shown to be crucially dependent on $F_{c}(\varepsilon)$, with the noise suppression (caused by Coulomb interactions) different for different injections. The derived energyresolved shot-noise suppression formulas indicate the possibility to probe the injection energy profile of a ballistic emitter in shot-noise measurements, thereby obtaining an important information not otherwise available from timeaveraged conductance measurements. On the other hand, that 
information may help to optimize the injection energy distribution to achieve the desired noise-suppression level.

The paper is organized as follows. In Sec. II we introduce the basic equations that describe the space-charge-limited ballistic transport: the collisionless kinetic equation coupled self-consistently with the Poisson equation. The obtained solutions allow us to find the analytical formulas for the mean current and the current fluctuation transmission expressed through the injection distribution function $F_{c}(\varepsilon)$. The shotnoise suppression factor is calculated for some particular cases in Sec. III. Finally, Sec. IV summarizes the main contributions of the paper, whereas in the appendix we present mathematical details concerning the derivation of the selfconsistent potential fluctuations.

\section{TRANSPORT AND NOISE IN SPACE-CHARGE- LIMITED BALLISTIC CONDUCTORS}

\section{A. The physical model}

Consider a two-terminal semiconductor ballistic sample with plane-parallel heavily doped contacts at $x=0$ and $x=l$. The structure may be considered as a $n-i-n$ heterodiode $^{7}$ operating under a space-charge-limited current regime in which the current is determined by a charge injection from the contacts rather than by intrinsic carriers of the ballistic region. The applied bias $U$ between the contacts is assumed to be fixed by a low-impedance external circuit. In order to simplify the problem, we assume that due to the large difference in the carrier density between the contacts and the sample, and hence in the corresponding Debye screening lengths, all the band bending occurs in the ballistic base, and therefore the relative position of the conduction band and the Fermi level $\varepsilon_{c}-\varepsilon_{F}$ does not change in the contacts. For such a modeling, all of the potential drop takes place exclusively inside the ballistic base and the contacts are excluded from the consideration. ${ }^{5-7}$ In contrast to Refs. 5-7, the injected carriers are not restricted to follow a thermal equilibrium distribution, their distribution is an arbitrary function determined by the particular properties of the emitter. Assuming the transversal size of the conductor sufficiently thick and high enough electron density, the electrostatic problem may be considered in a one-dimensional plane geometry. ${ }^{7}$

\section{B. Distribution function and its fluctuation in a self-consistent field}

A semiclassical ballistic transport is described by the collisionless kinetic equation for the time-dependent distribution function $\widetilde{F}\left(x, k_{x}, t\right)$ coupled self-consistently with the Poisson equation for the electrostatic potential $\tilde{\varphi}(x, t)$,

$$
\begin{gathered}
\left(\frac{\partial}{\partial t}+\frac{\hbar k_{x}}{m} \frac{\partial}{\partial x}+q \frac{d \tilde{\varphi}}{d x} \frac{\partial}{\hbar \partial k_{x}}\right) \tilde{F}\left(x, k_{x}, t\right)=0, \\
\frac{\partial^{2} \tilde{\varphi}}{\partial x^{2}}=\frac{q}{\kappa} \int \tilde{F}\left(x, k_{x}, t\right) \frac{\hbar d k_{x}}{\sqrt{2 m}},
\end{gathered}
$$

where $\kappa$ is the dielectric permittivity, and $m$ the electron effective mass. Since during the ballistic motion only the longitudinal momentum $k_{x}$ may vary, we use the electron distribution function averaged over the transversal momentum $\mathbf{k}_{\perp}$ according to

$$
\widetilde{F}\left(x, k_{x}, t\right)=\frac{\sqrt{2 m}}{\hbar} \int \frac{d \mathbf{k}_{\perp}}{(2 \pi)^{d}} f\left(x, k_{x}, \mathbf{k}_{\perp}, t\right),
$$

where $d$ is the dimension of a momentum space and $f\left(x, k_{x}, \mathbf{k}_{\perp}, t\right)$ is the occupation number of a quantum state at the cross section $x$. The additional multiplication factor $\sqrt{2 m} / \hbar$ in the integral (3) is introduced for further normalization convenience. Under the space-charge-limited transport conditions, the distribution function $\widetilde{F}$ and the space charge in the Poisson equation (2) are determined by the electrons injected from the contact. Due to the stochastic nature of the injection, the distribution function $\widetilde{F}\left(x, k_{x}, t\right)$ $=F\left(x, k_{x}\right)+\delta F\left(x, k_{x}, t\right)$ and the potential $\tilde{\varphi}(x, t)=\varphi(x)$ $+\delta \varphi(x, t)$ fluctuate in time around their time-averaged values. The nonuniform distribution of the injected carriers creates the potential minimum $\tilde{\varphi}_{m}(t)$ at a position $x=x_{m}$, which also fluctuates. It is the potential minimum fluctuations, that leads to the suppression of the injected current fluctuations. ${ }^{7}$ We assume that the applied bias is much larger than the characteristic energy spreading of injected electrons, so that the current injection from the second (receiving) contact is negligible. Another assumption is $U_{m} \ll U<U_{c r}$, where $U_{m} \equiv-\varphi_{m}$, and $U_{c r}$ is the bias at which the potential barrier vanishes. ${ }^{20}$ This assumption may be fulfilled under the condition of a strong screening that corresponds to the so-called "virtual cathode" approximation, when the potential minimum is so close to the contact, that one can disregard the region between the contact and the minimum. ${ }^{7}$ In this limit, only those electrons that are able to pass over the fluctuating barrier (transmitted electrons), contribute to the current and noise.

It is advantageous to use as a variable in the equations, instead of the kinetic energy, the total energy $\epsilon$ $=\hbar^{2} k_{x}^{2} /(2 m)-\Phi(x)$, where $\Phi(x) \equiv q \varphi(x)-q \varphi_{m}$ is the mean potential referenced to the minimum. By such a definition, $\Phi(x)>0$ in all the region (which is convenient for further consideration), whereas the potential energy $-\Phi(x)$ is negative. Equation (1), for the stationary case $(\partial / \partial t=0)$, in terms of these variables may be written as $(\partial / \partial x) F(x, \epsilon)$ $=0$. Its solution, being invariant on $x$, is expressed simply through the distribution function at the injecting contact $F_{c}$

$$
F(\epsilon)=F_{c}\left(\epsilon+\Phi_{c}\right) \theta(\epsilon),
$$

where $\Phi_{c} \equiv \Phi(0)$ is the potential at the contact, and the Heaviside step function $\theta(\epsilon)$ establishes the lower bound for the transmitted electrons. The fluctuation $\delta F$ is found from linearization of Eq. (1) around the mean values. Equivalently, one may just perturb the steady-state solution (4) as a compound function, and get

$$
\begin{aligned}
\delta F(\epsilon)= & \delta F_{c}\left(\epsilon+\Phi_{c}\right) \theta(\epsilon)+\frac{\partial F_{c}\left(\epsilon+\Phi_{c}\right)}{\partial \epsilon}\left(\delta \epsilon+\delta \Phi_{c}\right) \theta(\epsilon) \\
& +F_{c}\left(\epsilon+\Phi_{c}\right) \frac{\partial \theta(\epsilon)}{\partial \epsilon} \delta \epsilon .
\end{aligned}
$$


Taking into account that the perturbation of the total energy $\epsilon$ is related to the perturbation of the potential by $\delta \epsilon=$ $-\delta \Phi_{x}$ and using the property $\partial \theta(\epsilon) / \partial \epsilon=\delta(\epsilon)$, one finally obtains $^{21}$

$$
\begin{aligned}
\delta F(\epsilon)= & \delta F_{c}\left(\epsilon+\Phi_{c}\right) \theta(\epsilon)-F_{c}\left(\Phi_{c}\right) \delta \Phi_{x} \delta(\epsilon) \\
& +\frac{\partial F_{c}\left(\epsilon+\Phi_{c}\right)}{\partial \epsilon}\left(\delta \Phi_{c}-\delta \Phi_{x}\right) \theta(\epsilon) .
\end{aligned}
$$

The self-consistent potential fluctuations are defined as $\delta \Phi_{x} \equiv q \delta \varphi(x)-q \delta \varphi_{m}, \delta \Phi_{c} \equiv \delta \Phi_{0}$. This means that $\delta \Phi_{x}$ is measured in a frame referenced to the fluctuating potential minimum ( $\delta \Phi_{x_{m}}=0$ ). It is clear, that in such a consideration the contact potential and its fluctuation are related to the potential barrier height according to $\Phi_{c}=q U_{m}, \delta \Phi_{c}$ $=q \delta U_{m}$.

Equations (4) and (6) should now be substituted into the Poisson equations for $\Phi(x)$ and $\delta \Phi_{x}$, correspondingly, to find the self-consistent potential profile and its fluctuation.

\section{Steady state}

First we find the mean electron density as a function of the potential $\Phi$ by integrating $F$ over the momentum $k_{x}$ and changing the variable of integration $d k_{x}$ $=(\sqrt{2 m} / \hbar)(d \epsilon / 2 \sqrt{\epsilon+\Phi})$, we obtain

$$
N(\Phi)=\int_{0}^{\infty} F_{c}\left(\epsilon+\Phi_{c}\right) \frac{d \epsilon}{2 \sqrt{\epsilon+\Phi}} .
$$

Then, we solve the Poisson equation $d^{2} \Phi / d x^{2}$ $=\left(q^{2} / \kappa\right) N(\Phi)$, subject to the boundary conditions at the minimum $\Phi\left(x_{m}\right)=0$, and at the receiving contact $\Phi_{l}$ $\equiv \Phi(l)=q\left(U+U_{m}\right)$. First integration leads to the electricfield distribution

$$
E(\Phi)=-\frac{1}{q} \frac{d \Phi}{d x}=-\sqrt{\frac{2 q}{\kappa}} \sqrt{h(\Phi)},
$$

where

$$
\begin{aligned}
& h(\Phi)=\int_{0}^{\Phi} N(\widetilde{\Phi}) d \widetilde{\Phi}=\int_{0}^{\infty} F_{c}\left(\epsilon+\Phi_{c}\right)(\sqrt{\epsilon+\Phi}-\sqrt{\epsilon}) d \epsilon \\
&=\mathcal{F}_{0} \sqrt{\Phi}-\mathcal{F}_{1}+\frac{\mathcal{F}_{2}}{2 \sqrt{\Phi}}+O\left(\frac{1}{\Phi^{3 / 2}}\right), \Phi \rightarrow \infty \\
& \mathcal{F}_{j}\left(\Phi_{c}\right)=\int_{0}^{\infty} F_{c}\left(\epsilon+\Phi_{c}\right) \epsilon^{j / 2} d \epsilon, \quad j=0,1,2, \ldots
\end{aligned}
$$

The similar expansion for the electron density is given by

$$
N(\Phi)=\frac{d}{d \Phi} h(\Phi)=\frac{\mathcal{F}_{0}}{2 \sqrt{\Phi}}-\frac{\mathcal{F}_{2}}{4 \Phi^{3 / 2}}+O\left(\frac{1}{\Phi^{5 / 2}}\right)
$$

Integration of Eq. (8) with the expansion (9) yields at $x_{m}$ $\ll x \lesssim l$

$$
\Phi^{3 / 2}\left[1+\frac{3 \mathcal{F}_{1}}{\mathcal{F}_{0}} \frac{1}{\sqrt{\Phi}}\right] \approx \frac{9}{8} \frac{q^{2} \mathcal{F}_{0}}{\kappa}\left(x-x_{m}\right)^{2} .
$$

This equation, taken at $x=l$, may then be used to find the mean current

$$
\begin{aligned}
I & =\frac{q A}{\sqrt{2 m}} \int_{0}^{\infty} F_{c}\left(\epsilon+\Phi_{c}\right) d \epsilon=\frac{q A}{\sqrt{2 m}} \mathcal{F}_{0} \\
& \approx \frac{4}{9} \kappa A \sqrt{\frac{2 q}{m}} \frac{\left(U+U_{m}\right)^{3 / 2}}{\left(l-x_{m}\right)^{2}}\left[1+\frac{3 \mathcal{F}_{1}}{\mathcal{F}_{0}} \frac{1}{\sqrt{q\left(U+U_{m}\right)}}\right],
\end{aligned}
$$

where $A$ is the cross-sectional area. Here, the leading factor $\sim U^{3 / 2}$ (if one neglects $x_{m}, U_{m}$ in respect to $l, U$, respectively) is the Child current, which corresponds to what would be expected if all the electrons are injected with zero initial velocity. It is independent of the injection, but it is a function of the applied bias $U$, the length $l$, and the parameters of the material (the dielectric permittivity $\kappa$, the effective mass $m$ ). The next-order term $\sim U$ contains information about the injection distribution function and gives the correction due to the spread of electron momenta at the minimum, since $\mathcal{F}_{1} / \mathcal{F}_{0}=(\hbar / \sqrt{2 m})\left\langle k_{x}^{2}\right\rangle /\left\langle k_{x}\right\rangle$, where we denote the average values at the minimum by angular brackets. For the case of the Maxwellian injection, $F_{c}(\epsilon) \propto \exp \left(-\epsilon / k_{B} T\right)$, this ratio becomes $\mathcal{F}_{1} / \mathcal{F}_{0}=\sqrt{\pi k_{B} T} / 2$, and formula (13) leads to the Langmuir formula for a vacuum diode. , $22^{2}$

From Eq. (12), one can get the asymptotic formula for the potential profile $\varphi^{3 / 2}(x)=\frac{9}{4} \sqrt{m / 2 q}(I / \kappa A) x^{2}$. Substituting the Child current, one obtains the universal behavior $\varphi(x)$ $=U(x / l)^{4 / 3}$, at $x_{m} \ll x \leqslant l$, independently of the injection. The other quantities of interest tend to the following distributions: $\quad E(x)=-\frac{4}{3}(U / l)(x / l)^{1 / 3}, \quad N(x)=\frac{4}{9}\left(\kappa U / q l^{2}\right)(x /$ $l)^{-2 / 3}$. It is seen, that the time-averaged quantities, such as the mean current and the spatial profiles, asymptotically at high biases, are nonsensitive to the injection distribution function. (Electrons coming to the receiving contact with the energies much higher than their injecting energies forget about their initial spreading.) The injection distribution gives just a small correction to the lower-order terms, which however may be essential at intermediate biases. In contrast, the current noise is sensitive to the injection distribution in the leading-order terms, which decrease with bias, as will be demonstrated below.

\section{Current fluctuations}

The current fluctuation is obtained by integrating over the energy the fluctuation of the distribution function (6)

$$
\begin{aligned}
\delta I & =\frac{q A}{\sqrt{2 m}} \int_{0}^{\infty} \delta F_{c}\left(\epsilon+\Phi_{c}\right) d \epsilon-\frac{q A}{\sqrt{2 m}} F_{c}\left(\Phi_{c}\right) \delta \Phi_{c} \\
& \equiv \int_{0}^{\infty} \delta I_{c}\left(\epsilon+\Phi_{c}\right) d \epsilon+\delta I_{\text {Coul }} .
\end{aligned}
$$

Here, $\delta I_{c}(\epsilon)$ is the partial injected current fluctuation in a unit of energy. The last term $\delta I_{\text {Coul }}$, which is the current fluctuation caused by the long-range Coulomb interactions, may also be expressed more generally as $\delta I_{\text {Coul }}$ $=\left(\partial I / \partial \Phi_{c}\right) \delta \Phi_{c}=\left(\partial I / \partial U_{m}\right) \delta U_{m}$, reflecting the modulation effect of the potential barrier fluctuations. To find that term, 
we need to obtain $\delta \Phi_{c}$ as a function of the injected fluctuations $\delta F_{c}$, by solving the Poisson equation.

Integrating Eq. (6) over the momentum $k_{x}$, one gets the electron-density fluctuation as a sum of two contributions, $\delta N=\delta N^{i n j}+\delta N^{i n d}$, where the injected part

$$
\delta N^{i n j}(\Phi)=\int_{0}^{\infty} \delta F_{c}\left(\epsilon+\Phi_{c}\right) \frac{d \epsilon}{2 \sqrt{\epsilon+\Phi}},
$$

and the induced part

$$
\begin{aligned}
\delta N^{i n d}(\Phi)= & \left(\delta \Phi_{c}-\delta \Phi_{x}\right) \int_{0}^{\infty} \frac{\partial F_{c}\left(\epsilon+\Phi_{c}\right)}{\partial \epsilon} \frac{d \epsilon}{2 \sqrt{\epsilon+\Phi}} \\
& -\delta \Phi_{x} \frac{F_{c}\left(\Phi_{c}\right)}{2 \sqrt{\Phi}} \\
= & \frac{d N}{d \Phi} \delta \Phi_{x}-\left(\frac{d N}{d \Phi}+\frac{F_{c}\left(\Phi_{c}\right)}{2 \sqrt{\Phi}}\right) \delta \Phi_{c} .
\end{aligned}
$$

Substitution to the Poisson equation yields

$$
\begin{aligned}
\hat{L} \delta \Phi_{x} & \equiv\left[\frac{d^{2}}{d x^{2}}-\frac{q^{2}}{\kappa} \frac{d N}{d \Phi}\right] \delta \Phi_{x} \\
& =-\frac{q^{2}}{\kappa}\left(\frac{d N}{d \Phi}+\frac{F_{c}\left(\Phi_{c}\right)}{2 \sqrt{\Phi}}\right) \delta \Phi_{c}+\frac{q^{2}}{\kappa} \delta N^{i n j}(\Phi) .
\end{aligned}
$$

By solving this equation with the boundary conditions $\delta \Phi_{x_{m}}=0, \delta \Phi_{l}=\delta \Phi_{c}$ (see the appendix) we find the Coulomb correlation term in the form

$$
\delta I_{\text {Coul }}=-\int_{0}^{\infty} \delta I_{c}\left(\epsilon+\Phi_{c}\right)\left[1-\frac{3}{\sqrt{q U}}\left(\sqrt{\epsilon}-\frac{N_{m}}{F_{c}\left(\Phi_{c}\right)}\right)\right] d \epsilon,
$$

where $N_{m}$ is the electron density at the potential minimum. Substitution of the found expression for $\delta I_{\text {Coul }}$ into Eq. (14) for the total current fluctuation shows, that the leading-order terms, which do not depend explicitly on bias, are canceled, i.e., the injected current fluctuation is suppressed. The remaining contribution

$$
\delta I=\frac{3}{\sqrt{q U}} \int_{0}^{\infty}\left[\sqrt{\epsilon}-\frac{N_{m}}{F_{c}\left(\Phi_{c}\right)}\right] \delta I_{c}\left(\epsilon+\Phi_{c}\right) d \epsilon
$$

is $\propto U^{-1 / 2}$. We rewrite this expression in the form

$$
\delta I=\int_{\Phi_{c}}^{\infty} \gamma(\varepsilon) \delta I_{c}(\varepsilon) d \varepsilon
$$

in which the effect of the interactions is summarized by the quantity $\gamma(\varepsilon)$ determined by

$$
\gamma(\varepsilon)=\frac{3}{\sqrt{q U}}\left[\sqrt{\varepsilon-\Phi_{c}}-v\left(\Phi_{c}\right)\right],
$$

and the introduced energy $\varepsilon=\epsilon+\Phi_{c}$ corresponds to the (longitudinal) kinetic energy of electrons at the injecting contact. The constant $v$ in Eq. (21) is the characteristic velocity given by

$$
v\left(\Phi_{c}\right)=\frac{N_{m}}{F_{c}\left(\Phi_{c}\right)}=\frac{1}{F_{c}\left(\Phi_{c}\right)} \int_{\Phi_{c}}^{\infty}\left[-\frac{\partial F_{c}}{\partial \varepsilon}\right] \sqrt{\varepsilon-\Phi_{c}} d \varepsilon .
$$

The main result, which follows from the derived expression (21), is that $\gamma(\varepsilon)$ is a decreasing function of the applied bias $U$. With higher bias, a larger suppression of the current fluctuations is expected. Another important conclusion is that the suppression effect is different for different injection shapes $F_{c}(\varepsilon)$. The dependence on $F_{c}(\varepsilon)$ is summarized by the characteristic velocity $v$ determined by Eq. (22). Note, that the function $\gamma(\varepsilon)$ has a meaning of the current fluctuation transfer function, ${ }^{7}$ and in general may be as positive, as negative, depending on the particular energy $\varepsilon$. In the absence of correlations, $\gamma^{\text {uncor }}(\varepsilon)=\theta\left(\varepsilon-q U_{m}\right)$, that means the fluctuations of all energies above the barrier height $q U_{m}$ are equally transmitted.

Having found the current fluctuation $\delta I$ expressed through the injected current fluctuations $\delta I_{c}(\epsilon)$, the currentnoise spectral density may then be obtained from Eq. (20) as

$$
S_{I} \Delta f=\int_{\Phi_{c}}^{\infty} \int_{\Phi_{c}}^{\infty} \gamma(\varepsilon) \gamma\left(\varepsilon^{\prime}\right)\left\langle\delta I_{c}(\varepsilon) \delta I_{c}\left(\varepsilon^{\prime}\right)\right\rangle d \varepsilon d \varepsilon^{\prime} .
$$

Here, $\Delta f$ is the frequency bandwidth (we assume the lowfrequency limit), and in such a presentation the function $\gamma(\varepsilon)$ plays the role of the energy resolved shot-noisesuppression factor.

The incoming electrons may be correlated in energy $a$ priori due to the properties of an emitter. In general case of non-Poissonian injection, one can define the shot-noisesuppression factor due to a pure Coulomb suppression by

$$
\Gamma_{C}=\frac{\int_{\Phi_{c}}^{\infty} \int_{\Phi_{c}}^{\infty} \gamma(\varepsilon) \gamma\left(\varepsilon^{\prime}\right)\left\langle\delta I_{c}(\varepsilon) \delta I_{c}\left(\varepsilon^{\prime}\right)\right\rangle d \varepsilon d \varepsilon^{\prime}}{\int_{\Phi_{c}}^{\infty} \int_{\Phi_{c}}^{\infty}\left\langle\delta I_{c}(\varepsilon) \delta I_{c}\left(\varepsilon^{\prime}\right)\right\rangle d \varepsilon d \varepsilon^{\prime}},
$$

which can be easily found when the properties of injected carriers are given.

For the particular case when the injected carriers of different energies are uncorrelated,

$$
\left\langle\delta I_{c}(\varepsilon) \delta I_{c}\left(\varepsilon^{\prime}\right)\right\rangle=K(\varepsilon)(\Delta f) \delta\left(\varepsilon-\varepsilon^{\prime}\right),
$$

the shot-noise-suppression factor (24) is simplified to

$$
\Gamma_{C}=\frac{\int_{\Phi_{c}}^{\infty} \gamma^{2}(\varepsilon) K(\varepsilon) d \varepsilon}{\int_{\Phi_{c}}^{\infty} K(\varepsilon) d \varepsilon} .
$$

Furthermore, for the Poissonian injection, the property of the kernel $K$ is such that $K(\varepsilon) \propto I_{c}(\varepsilon) \propto F_{c}(\varepsilon)$. Hence, one can find

$$
\Gamma_{\text {Poisson }}=\frac{\int_{\Phi_{c}}^{\infty} \gamma^{2}(\varepsilon) F_{c}(\varepsilon) d \varepsilon}{\int_{\Phi_{c}}^{\infty} F_{c}(\varepsilon) d \varepsilon} \rightarrow \frac{S_{I}}{2 q I} .
$$


Depending on the injection, one of the expressions (24), (26), and (27) can be used together with the function $\gamma(\varepsilon)$ given by Eq. (21) to evaluate the shot-noise-suppression level in ballistic space-charge-limited conductors under the action of Coulomb interactions. Note that the formula for $\gamma(\varepsilon)$ is valid for any given energy distribution and statistical properties of the injected carriers under the condition of a high bias, that is $U \gg U_{m}$ and $U$ much larger than the characteristic energy spreading of injected electrons. The upper bound for the bias is however restricted by the condition of the existence of the potential barrier $U<U_{c r}$ (space-chargelimited transport). Both conditions may be fulfilled simultaneously under a sufficiently strong screening, i.e., the length of the conductor should be much larger than the characteristic screening length. ${ }^{7}$

\section{EXAMPLES}

To illustrate the implementation of the results, we consider some examples. For the Maxwell-Boltzmann (MB) injection distribution (nondegenerate equilibrium electron gas is injected) we obtain $v=\sqrt{\pi k_{B} T} / 2$, i.e., it only depends on the temperature of the injected electrons, but otherwise is independent of the material parameters, since its dependence on the barrier height is canceled out. For this case, Eq. (21) gives

$$
\gamma_{M B}(\varepsilon)=3 \sqrt{\frac{k_{B} T}{q U}}\left(\sqrt{\frac{\varepsilon-\Phi_{c}}{k_{B} T}}-\frac{\sqrt{\pi}}{2}\right),
$$

which coincides with the formula derived by North. ${ }^{7,23}$ The corresponding shot-noise-suppression factor follows from Eq. (27)

$$
\Gamma_{M B}=9\left(1-\frac{\pi}{4}\right) \frac{k_{B} T}{q U} .
$$

For a quantitative estimation consider the heterodiode with GaAs contacts and an $\mathrm{Al}_{0.05} \mathrm{Ga}_{0.95} \mathrm{As}$ ballistic base. ${ }^{24}$ For the contact doping $4 \times 10^{16} \mathrm{~cm}^{-3}$ at $T=50 \mathrm{~K}$, we obtain the injected electron density about $7.25 \times 10^{14} \mathrm{~cm}^{-3}$, which corresponds to the Debye screening length $L_{D}=46 \mathrm{~nm}$. Then for the $1.5 \mu$-length diode and $U \approx 45 k_{B} T / q$, the noisesuppression level estimated from the exact solutions ${ }^{24}$ gives $\Gamma_{M B} \approx 0.04$, which is close to the value calculated from the asymptotic formula (29).

Now we shall demonstrate, that the shot-noisesuppression level may be achieved even deeper than that given by Eq. (29) for the MB case, without involving any other correlations (like the Pauli exclusion principle) in addition to the Coulomb correlations. The higher suppression may be achieved by modifying the energy profile for the injected carriers. Consider the heterodiode under the same set of parameters considered above, in which, in addition to the Maxwell-Boltzmann injection, nonequilibrium carriers are injected from a specially designed emitter, so that the injected distribution function has an additional peak at the energy $\varepsilon_{0}$ [see inset of Fig. 1(b)]. According to our theory, these additional electrons do not change the current-voltage characteristics much. Its asymptotic behavior is again the Child law. However, the noise properties change significantly depending on the parameters of the electron-energy

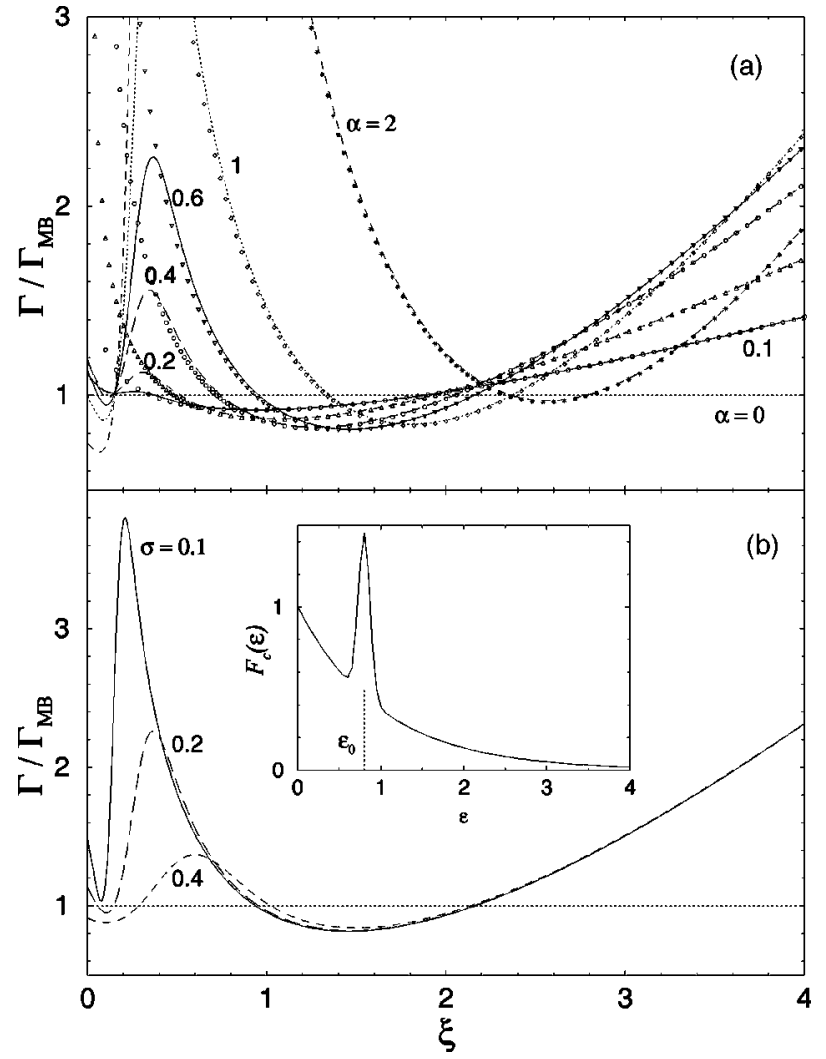

FIG. 1. The shot-noise suppression level $\Gamma$ (caused by Coulomb interactions) for the Maxwell-Boltzmann injection with an additional peak at $\varepsilon=\varepsilon_{0}$ (shown in the inset) with respect to the case when no peak is present. The ratio $\Gamma / \Gamma_{M B}$ is shown as a function of the peak position $\xi=\left(\varepsilon_{0}-\Phi_{c}\right) /\left(k_{B} T\right)$. (a) the peak parameter $\alpha$ is varied. The results are compared for two different shapes of the peak: $\delta$ function given by Eq. (30) (symbols); Gaussian function given by Eq. (33) for $\sigma=0.2$ (lines). (b) the Gaussian peak case: the width of the peak $\sigma$ is varied, while $\alpha=0.6$ is fixed.

peak, its magnitude, position, etc. In particular, the noisesuppression level may be obtained lower or higher than the MB shot noise by simply shifting the position of the peak (of the emitter) in respect to the potential barrier.

Let us assume that the width of the peak is narrow on the scale of the temperature $T$. For simplicity, we model it first by a $\delta$ function (monoenergetic electrons)

$$
F_{c}(\varepsilon) \propto e^{-\varepsilon / k_{B} T}+\tilde{\alpha} k_{B} T \delta\left(\varepsilon-\varepsilon_{0}\right) .
$$

The injected carriers, from both the Maxwellian tail and the peak, are assumed to be uncorrelated, so that Eq. (27) can be applied. Thus, for the distribution (30) one gets the (normalized) characteristic velocity (22) as

$$
w \equiv \frac{v}{\sqrt{k_{B} T}}=\frac{\sqrt{\pi}}{2}\left(1+\frac{\alpha}{\sqrt{\pi \xi}}\right),
$$

where $\alpha=\tilde{\alpha} e^{\Phi_{c}}$ is the ratio between the two currents: from the $\delta$ peak and from the MB exponential tail, and $\xi=\left(\varepsilon_{0}\right.$ $\left.-\Phi_{c}\right) /\left(k_{B} T\right)$ is the dimensionless position of the peak. The shot-noise-suppression factor is then obtained as (below we omit the subindex $c$ at $\Gamma$ ) 


$$
\Gamma=9 \frac{k_{B} T}{q U} \frac{1-w \sqrt{\pi}+w^{2}+\alpha(\sqrt{\xi}-w)^{2}}{1+\alpha} .
$$

In the limit when the electron-energy peak vanishes, $\alpha \rightarrow 0$, formula (32) is reduced to the suppression factor (29) for the MB injection. We have studied how $\Gamma$ deviates from $\Gamma_{M B}$ when the peak current $\alpha$ and the peak position $\xi$ are varied. The results are illustrated in Fig. 1(a). The dependence of $\Gamma / \Gamma_{M B}$ on $\xi$ was found to be nonmonotonic displaying a minimum. In some range of $\xi$ around the minimum, $\Gamma / \Gamma_{M B}<1$, that means the additional electron-energy peak at the injecting contact results in a less noisy transmission, than in the case of its absence. The minimal noise is observed for $\alpha \approx 0.6$, for which we find $\Gamma / \Gamma_{M B} \approx 0.814$ at $\xi_{\text {min }} \approx 1.45$. As follows from Fig. 1(a), the most effective noise suppression occurs when the peak is about $1-2 k_{B} T$ above the barrier. When it is higher in energy, or too close to the barrier position $\left(\varepsilon_{0}-\Phi_{c} \lesssim k_{B} T\right)$, the noise is enhanced in respect to the MB case (although it may still be below the Poissonian value).

The analysis for other shapes of the peak shows that the results are similar to those for the $\delta$ peak. As an example, we present here the results for the case when the peak is modeled by the Gaussian distribution function

$$
F_{c}(\varepsilon) \propto e^{-\varepsilon / k_{B} T}+\varrho e^{-\left(\varepsilon-\varepsilon_{0}\right)^{2} /\left(\sigma k_{B} T\right)^{2}},
$$

where the factor $\varrho$ is defined by $\varrho=2 \tilde{\alpha} /\{\sigma \sqrt{\pi}[1$ $\left.\left.+\operatorname{erf}\left(\varepsilon_{0} / \sigma k_{B} T\right)\right]\right\}$. By such a definition, the parameter $\alpha$ $=\tilde{\alpha} e^{\Phi_{c}}$ gives again, as in the previous case, the ratio between the current originated from the Gaussian peak and that from the MB tail. A comparison between the two cases is presented in Fig. 1(a). It is seen, that at high values of $\xi$ the results for the noise suppression for both cases of the Gaussian and $\delta$ peak coincide. It can be shown, that this occurs at $\xi \gtrsim 5 \sigma$. Hence, when the peak width $\sigma<\left(\xi_{\min } / 5\right) \approx 0.3$, the minimal noise occurs at the same peak position $\xi_{\text {min }}$, independently of the value of $\sigma$. The information on the peak width is presented, however, in the noise-suppression curves at low values of $\xi$. While $\Gamma$ for the $\delta$-peak case diverges at $\xi \rightarrow 0$, due to a singularity of the $\delta$ function, the noisesuppression factor for the Gaussian-peak case exhibits a local maximum [see Figs. 1(a) and 1(b)]. The magnitude of this noise enhancement (in respect to the MB case) depends on $\sigma$ : the narrower the peak, the larger the noise enhancement and the closer is the location of the maximum to the potential barrier energy [see Fig. 1(b)].

Summarizing this example, to observe the lower noise level for nondegenerate ballistic electrons, the additional (to the MB tail) electrons should be injected with the energy about $1.45 k_{B} T$ above the potential barrier. This value is independent of the energy spreading of the "peak" electrons once the latter is less than $0.3 k_{B} T$. The optimal ratio between the current from the "peak" electrons and the MB electrons is about 0.6 .

It is seen, that the shot noise contains important information on: (i) the injection energy profile, and (ii) the parameters of the injected space charge, such as the potential barrier height and the electron density $N_{m}$ at the barrier position. Therefore, noise measurements may be used as a tool to study those characteristics.

\section{SUMMARY}

In conclusion, we have presented a self-consistent theory of transport and current noise in two-terminal ballistic spacecharge-limited conductors under the action of Coulomb interactions. We have derived the analytical formulas that account for the non-Poissonian injection with arbitrary distribution function and correlation properties of injected electrons, and these may be used to estimate: (i) the mean current beyond the Child approximation with a next-order term specific of the injection distribution function; (ii) the current-noise spectral density under the action of Coulomb interactions, which depends in the leading-order terms on the injection distribution function and decreases with bias; (iii) the noise-suppression factor in respect to the injected nonPoissonian electron flow. ${ }^{25}$

The obtained analytical formula for the energy-resolved shot-noise suppression may suggest a new field of investigation on the optimization of the injection energy profile to achieve the desired noise-suppression level. The presented examples clearly show, that the noise-suppression level may be controlled by monitoring the injection energy profile.

The sensitivity of the noise-suppression level to the injection parameters opens up new perspectives in shot-noise measurements as a tool not only to identify the ballistic transport in mesoscopic conductors, but also to reveal an important information on the injection energy profile and the level of Coulomb interactions in the structure. Experiments have succeeded recently in observing shot noise in ballistic quantum point contacts ${ }^{26,27}$ and some other mesoscopic systems (see, e.g., Refs. 28-30). We believe, that it would be similarly possible to measure the shot noise in space-chargelimited ballistic conductors.

Additionally, it is important to emphasize the difference between the asymptotic behavior of the shot noise in diffusive and ballistic systems under the presence of a space charge. In the former case the noise-suppression level is limited by the constant, specific of the dominating scattering mechanism, ${ }^{31-34}$ while in the latter the suppression may be arbitrarily strong, which may be important from the point of view of possible applications.

\section{ACKNOWLEDGMENTS}

This work was partially supported by the Generalitat de Catalunya, Spain, and the NATO linkage grant HTECH.LG 974610.

\section{APPENDIX A: DERIVATION OF THE SELF-CONSISTENT POTENTIAL FLUCTUATIONS}

The second-order differential equation (17) with spatially dependent coefficients can be solved explicitly for $\delta \Phi_{x}{ }^{7,35}$ Here, we need just the value of $\delta \Phi_{c}$, which has entered explicitly into the nonhomogeneous part and can be obtained by applying the Green's theorem for the self-adjoint operator $\hat{L}$

$$
\begin{gathered}
\int_{x_{m}}^{l}\left[u(x) \hat{L} \delta \Phi_{x}-\delta \Phi_{x} \hat{L} u(x)\right] d x \\
=\left.\left(u(x) \frac{d \delta \Phi}{d x}-\delta \Phi_{x} \frac{d u}{d x}\right)\right|_{x_{m}} ^{l} .
\end{gathered}
$$


It is convenient to chose the function $u(x)$ as a solution of the homogeneous equation $\hat{L} u(x)=0$ satisfying the boundary condition $u(l)=0$. This gives

$$
\begin{aligned}
& -\delta \Phi_{c} \frac{q^{2}}{\kappa} \int_{x_{m}}^{l} u\left(\frac{d N}{d \Phi}+\frac{F_{c}\left(\Phi_{c}\right)}{2 \sqrt{\Phi}}\right) d x+\frac{q^{2}}{\kappa} \int_{x_{m}}^{l} u \delta N^{i n j} d x \\
& =-u^{\prime}(l) \delta \Phi_{c}-u\left(x_{m}\right) \delta \Phi_{x_{m}}^{\prime},
\end{aligned}
$$

where prime stands for the derivative on $x$. It can be shown, that at large $U$, both terms in the right-hand side of Eq. (A2) may be neglected. Indeed, ${ }^{36} u^{\prime}(l)=1 / E(l)=O\left(\Phi_{l}^{-1 / 4}\right) \rightarrow 0$, at $\Phi_{l} \rightarrow \infty$. The term $u\left(x_{m}\right) \delta \Phi_{x_{m}}^{\prime}$ may be evaluated from the matching with the expression similar to Eq. (A2) for the adjacent region $0<x<x_{m}$. It occurs to be $O(1)$ at $\Phi_{l} \rightarrow \infty$, and hence gives negligible contribution in respect to the leading terms $O\left(\Phi_{l}^{3 / 4}\right)$ (see below). Changing the variable of integration $d x=-d \Phi /(q E)$, one gets

$$
\delta \Phi_{c} \int_{0}^{\Phi_{l}} \frac{u}{E}\left(\frac{d N}{d \Phi}+\frac{F_{c}\left(\Phi_{c}\right)}{2 \sqrt{\Phi}}\right) d \Phi=\int_{0}^{\Phi_{l}} \frac{u}{E} \delta N^{i n j} d \Phi .
$$

In this equation the integrals may be integrated by parts in a similar way

$$
\begin{aligned}
\int_{0}^{\Phi_{l}} \frac{u}{E} \frac{d G_{i}}{d \Phi} d \Phi & =\left.\left(\frac{u}{E} G_{i}\right)\right|_{0} ^{\Phi_{l}}-\int_{0}^{\Phi_{l}} G_{i} \frac{d}{d \Phi}\left(\frac{u}{E}\right) d \Phi \\
& =\frac{1}{q} \int_{0}^{\Phi_{l}} \frac{G_{i}}{E^{3}} d \Phi, \quad i=1,2,
\end{aligned}
$$

with

$$
\begin{gathered}
G_{1}(\Phi)=N(\Phi)-N(0)+F_{c}\left(\Phi_{c}\right) \sqrt{\Phi}, \\
G_{2}(\Phi)=\int_{0}^{\infty} \delta F_{c}\left(\epsilon+\Phi_{c}\right)(\sqrt{\epsilon+\Phi}-\sqrt{\epsilon}) d \epsilon .
\end{gathered}
$$

Notice, that the first term in right-hand side of Eq. (A4) is zero, since at the upper limit $u\left(\Phi_{l}\right)=0$, and at the lower limit we have $G_{i}(\Phi) \sim \Phi, E(\Phi) \sim \sqrt{\Phi}$ at $\Phi \rightarrow 0$, and $u(0)$ $=\kappa /[q N(0)]$ is finite. In the second integral of Eq. (A4) we have used ${ }^{36}$

$$
\frac{d}{d \Phi}\left(\frac{u}{E}\right)=-\frac{1}{q E} \frac{d}{d x}\left(\frac{u}{E}\right)=-\frac{1}{q E^{3}} .
$$

Thus, Eq. (A3) becomes

$$
\begin{aligned}
& \delta \Phi_{c} \int_{0}^{\Phi_{l}} \frac{N_{m}-N(\Phi)-F_{c}\left(\Phi_{c}\right) \sqrt{\Phi}}{E^{3}(\Phi)} d \Phi \\
& \quad=\int_{0}^{\infty} d \epsilon \delta F_{c}\left(\epsilon+\Phi_{c}\right) \int_{0}^{\Phi_{l}} d \Phi \frac{\sqrt{\epsilon+\Phi}-\sqrt{\epsilon}}{E^{3}(\Phi)}
\end{aligned}
$$

where $N_{m} \equiv N(\Phi=0)$ is the electron density at the potential minimum. At the high-bias limit $\Phi_{l} \rightarrow \infty$, by using Eqs. (8)(11), one obtains

$$
\begin{gathered}
\int_{0} \frac{\Phi_{l} N_{m}-N(\Phi)-F_{c}\left(\Phi_{c}\right) \sqrt{\Phi}}{E^{3}(\Phi)} d \Phi \\
=\frac{4}{3} \Phi_{l}^{3 / 4} F_{c}\left(\Phi_{c}\right)\left[1+3\left(\frac{3 \mathcal{F}_{1}}{2 \mathcal{F}_{0}}-\frac{N_{m}}{F_{c}\left(\Phi_{c}\right)}\right) \Phi_{l}^{-1 / 2}\right. \\
\left.+O\left(\Phi_{l}^{-1}\right)\right], \\
\int_{0}^{\frac{\Phi_{l} \sqrt{\epsilon+\Phi}-\sqrt{\epsilon}}{E^{3}(\Phi)} d \Phi=\frac{4}{3} \Phi_{l}^{3 / 4}\left[1+3\left(\frac{3 \mathcal{F}_{1}}{2 \mathcal{F}_{0}}-\sqrt{\epsilon}\right) \Phi_{l}^{-1 / 2}\right.} \\
\left.+O\left(\Phi_{l}^{-1}\right)\right] .
\end{gathered}
$$

In the latter expansion it is assumed, that the range of valuable injection energies is much less than the applied bias, $\epsilon$ $\ll \Phi_{l}$. Substituting these expansions into Eq. (A8), one obtains

$F_{c}\left(\Phi_{c}\right) \delta \Phi_{c}$

$$
=\int_{0}^{\infty} \delta F_{c}\left(\epsilon+\Phi_{c}\right)\left[1-\frac{3}{\sqrt{\Phi_{l}}}\left(\sqrt{\epsilon}-\frac{N_{m}}{F_{c}\left(\Phi_{c}\right)}\right)\right] d \epsilon,
$$

which is used to find the Coulomb correlation term $\delta I_{\text {Coul }}$ in Eq. (14).
${ }^{1}$ M. J. M. de Jong and C. W. J. Beenakker, in Mesoscopic Electron Transport, edited by L. P. Kowenhoven, G. Schön, and L. L. Sohn (Kluwer, Dordrecht, 1997), p. 225.

${ }^{2}$ Ya.M. Blanter and M. Büttiker, cond-mat/9910158.

${ }^{3}$ R. Landauer, Nature (London) 392, 658 (1998).

${ }^{4}$ R. Landauer, Phys. Rev. B 47, 16427 (1993); Physica B 227, 156 (1996).

${ }^{5}$ T. González, O.M. Bulashenko, J. Mateos, D. Pardo, and L. Reggiani, Phys. Rev. B 56, 6424 (1997).

${ }^{6}$ O.M. Bulashenko, J. Mateos, D. Pardo, T. González, L. Reggiani, and J.M. Rubí, Phys. Rev. B 57, 1366 (1998).

${ }^{7}$ O.M. Bulashenko, J.M. Rubí, and V.A. Kochelap, Phys. Rev. B 61, 5511 (2000).
${ }^{8}$ C. W. J. Beenakker and H. van Houten, in Solid State Physics: Advances in Research and Applications, edited by H. Ehrenreich and D. Turnbull (Academic, San Diego, 1991), Vol. 44, p.1.

${ }^{9}$ K. Natori, J. Appl. Phys. 76, 4879 (1994).

${ }^{10}$ L.E. Wernersson, A. Litwin, L. Samuelson, and H. Xu, IEEE Trans. Electron Devices 44, 1829 (1997).

${ }^{11}$ F.G. Pikus and K.K. Likharev, Appl. Phys. Lett. 71, 3661 (1997); K.K. Likharev, Proc. IEEE 87, 606 (1999).

${ }^{12}$ G.R. Facer, B.E. Kane, A.S. Dzurak, R.J. Heron, N.E. Lumpkin, R.G. Clark, L.N. Pfeiffer, and K.W. West, Phys. Rev. B 59, 4622 (1999).

${ }^{13}$ W.J. Kaiser and L.D. Bell, Phys. Rev. Lett. 60, 1406 (1988).

${ }^{14}$ R. Heer, J. Smoliner, G. Strasser, and E. Gornik, Phys. Rev. B 59, 
4618 (1999).

${ }^{15}$ A.F.J. Levi, J.R. Hayes, P.M. Platzman, and W. Wiegmann, Phys. Rev. Lett. 55, 2071 (1985).

${ }^{16}$ M. Heiblum, M.I. Nathan, D.C. Thomas, and C.M. Knoedler, Phys. Rev. Lett. 55, 2200 (1985).

${ }^{17}$ A. Palevski, M. Heiblum, C.P. Umbach, C.M. Knoedler, A.N. Broers, and R.H. Koch, Phys. Rev. Lett. 62, 1776 (1989).

${ }^{18}$ Z. Xie and S.A. Lyon, Appl. Phys. Lett. 75, 2085 (1999).

${ }^{19}$ V.V. Mitin, V.A. Kochelap, and M.A. Stroscio, Quantum Heterostructures (Cambridge University Press, New York, 1999), Chap. 9.

${ }^{20}$ The case $U>U_{c r}$ is not interesting, since the transport is no longer space-charge limited, and the noise is no longer suppressed by Coulomb correlations.

${ }^{21}$ One should not confuse the notations $\delta \epsilon$ and $\delta(\epsilon)$. The former means a (small) variation in the total energy $\epsilon$, while the latter is the Dirac $\delta$ function on the argument $\epsilon$.

${ }^{22}$ A. van der Ziel, Noise (Prentice-Hall, Englewood Cliffs, NJ, 1954), Chaps. 5-1 and 14-2.

${ }^{23}$ D.O. North, RCA Rev. 4, 441 (1940).

${ }^{24}$ O.M. Bulashenko, J.M. Rubí, and V.A. Kochelap, Appl. Phys. Lett. 75, 2614 (1999).

${ }^{25}$ It should be mentioned, that the ballistic Fermi injection under the Coulomb interaction has been addressed by Y. Naveh, A.N. Korotkov, and K.K. Likharev, Phys. Rev. B 60, R2169 (1999). The authors present the transfer functions $\gamma$ in terms of the derivatives of the potential barrier height $\Phi_{0}$ over the partial injection currents $i_{S}(E)$, whereas $\Phi_{0}$ is a functional of $i_{S}(E)$. For the particular electrostatic configuration of a $2 \mathrm{D}$ conductor under a gate, those derivatives are computed numerically.

${ }^{26}$ M.I. Reznikov, M. Heiblum, H. Shtrikman, and D. Mahalu, Phys. Rev. Lett. 75, 3340 (1995)

${ }^{27}$ A. Kumar, L. Saminadayar, D.C. Glattli, Y. Jin, and B. Etienne, Phys. Rev. Lett. 76, 2778 (1996).

${ }^{28}$ A.H. Steinbach, J.M. Martinis, and M.H. Devoret, Phys. Rev. Lett. 76, 3806 (1996)

${ }^{29}$ R.J. Schoelkopf, P.J. Burke, A.A. Kozhevnikov, D.E. Prober, and M.J. Rooks, Phys. Rev. Lett. 78, 3370 (1997).

${ }^{30}$ X. Jehl, P. Payet-Burin, C. Baraduc, R. Calemczuk, and M. Sanquer, Rev. Sci. Instrum. 70, 2711 (1999).

${ }^{31}$ T. González, C. González, J. Mateos, D. Pardo, L. Reggiani, O.M. Bulashenko, and J.M. Rubí, Phys. Rev. Lett. 80, 2901 (1998).

${ }^{32}$ T. González, J. Mateos, D. Pardo, O.M. Bulashenko, and L. Reggiani, Phys. Rev. B 60, 2670 (1999).

${ }^{33}$ C.W.J. Beenakker, Phys. Rev. Lett. 82, 2761 (1999).

${ }^{34}$ H. Schomerus, E.G. Mishchenko, and C.W.J. Beenakker, Phys. Rev. B 60, 5839 (1999).

${ }^{35}$ O.M. Bulashenko, G. Gomila, J.M. Rubí, and V.A. Kochelap, Appl. Phys. Lett. 70, 3248 (1997); J. Appl. Phys. 83, 2610 (1998).

${ }^{36}$ The function $u(x)$ can be found through the second independent solution $E(x)$ of the homogeneous equation $\hat{L} E(x)=0$, with the boundary condition $E\left(x_{m}\right)=0$. Here, however, we do not need an explicit formula for $u$, employing just the relation $E u^{\prime}$ $-E^{\prime} u=W=1$, where $W$ is the Wronskian (Ref. 7). 